\title{
INTEGRALS OF WEIGHTED MAXIMAL LOGARITHMIC KERNELS ON BOUNDED VILENKIN GROUPS
}

\author{
ISTVÁN MEZŐ AND KÁROLY NAGY
}

\begin{abstract}
The integrals of maximal Riesz- and Nörlund kernels are infinite, so we have to use some weight function to "pull them back" to the finite. In this paper we give necessary and sufficient conditions for weight function to get finite integral on bounded Vilenkin groups. For our motivation we refer the readers to $[4,5,6]$.
\end{abstract}

\section{INTRODUCTION}

Kernel functions play an important role in harmonic analysis; this is the case in harmonic analysis on algebraic structures, too. The fundamental problem in Fourier theory is the convergence of the partial sums of Fourier series of functions in some spaces. According to the BanachSteinhaus Theorem, this problem is approachable by the integrals of kernel functions as one can see in [3].

In this article we investigate integrals of maximal Riesz- and Nörlund kernels on bounded Vilenkin groups. Since, these integrals are infinite, we use some weight function to "pull them back" to the finite.

Let us introduce the standard notations $[1,8]$.

$$
\mathbb{Z}_{m}:=\{0,1, \ldots, m-1\}
$$

represents the $m$ th cyclic group. Let us supply $\mathbb{Z}_{m}$ with the discrete topology and with the measure $\mu_{m}$ which is defined by $\mu_{m}(\{x\})=1 / m$ for each $x \in \mathbb{Z}_{m}$.

Let $\left(m_{k}: k \in \mathbb{N}\right)$ be a sequence of natural numbers, which members are greater than 1 . Let

$$
G_{m}:=\underset{k=0}{\times} \mathbb{Z}_{m_{k}}
$$

This set is an abelian group with coordinate-wise addition modulo $m_{k}$. With product topology and product measure $\mu$ the group $G_{m}$ is a compact topological space and probability space, called Vilenkin group. If $m_{*}:=\sup _{k \in \mathbb{N}} m_{k}<\infty$, we say that $G_{m}$ is bounded. In the present paper we deal with bounded Vilenkin groups, only. In special case $m_{k}=2$ for all $k \in \mathbb{N}$, it is called Walsh group.

2000 Mathematics Subject Classification. 42C10.

Key words and phrases. (Maximal-) Riesz- and Nörlund kernels, weighted kernels, logarithmic means, Vilenkin group, (hyper)harmonic numbers. 
The elements of $G_{m}$ can be represented by a sequence $x=\left(x_{i}: i \in\right.$ $\mathbb{N}$ ), where $x_{i} \in \mathbb{Z}_{m_{i}}\left(i \in \mathbb{N}\right.$ ). The group operation on $G_{m}$ (denoted by $+)$ is the coordinate-wise addition modulo $m_{k}$.

A base for the neighbourhoods of $G_{m}$ is given by

$$
I_{0}(x):=G_{m}, \quad I_{n}(x):=\left\{y \in G_{m} \mid y_{i}=x_{i} \text { if } i<n\right\} .
$$

Let $0:=(0: i \in \mathbb{N}) \in G_{m}$ denote the null element of $G_{m}$. By convenience, $I_{n}(0)$ is denoted by $I_{n}$.

Each natural number $n$ can be expressed uniquely in the form

$$
n=\sum_{i=0}^{\infty} n_{i} M_{i}
$$

where $n_{i} \in\left\{0,1, \ldots, m_{i}-1\right\}$ and $M_{0}:=1, M_{n+1}:=m_{n} M_{n}(n \in \mathbb{N})$. (For the Walsh group $M_{n}=2^{n}$.) Let the order of $n>0$ be defined by $|n|:=\max \left\{j \in \mathbb{N}: n_{j} \neq 0\right\}$. That is, $M_{|n|} \leq n<M_{|n|+1}$ holds.

The generalised Rademacher functions are defined as

$$
r_{k}(x)=\exp \left(2 \pi i \frac{x_{k}}{m_{k}}\right) \quad\left(x \in G_{m}, n \in \mathbb{N}, i=\sqrt{-1}\right) .
$$

By these functions a complete orthonormal system is constructed on Vilenkin group, called Vilenkin system:

$$
\psi_{n}(x):=\prod_{j=0}^{\infty}\left(r_{j}(x)\right)^{n_{j}} \quad\left(x \in G_{m}\right) .
$$

Each $\psi_{n}$ is a character of $G_{m}$, and all the characters of $G_{m}$ are of this form [9]. Define the $m$-adic addition as

$$
k \oplus n:=\sum_{j=0}^{\infty}\left(k_{j}+n_{j}\left(\bmod m_{j}\right)\right) M_{j} \quad(k, n \in \mathbb{N}) .
$$

Then, $\psi_{k \oplus n}=\psi_{k} \psi_{n}, \psi_{n}(x+y)=\psi_{n}(x) \psi_{n}(y), \psi(-x)=\bar{\psi}_{n}(x),\left|\psi_{n}\right|=1$ $\left(k, n \in \mathbb{N}, x, y \in G_{m}\right)$.

Now, we define the main notions of this paper.

The Fourier coefficients, Dirichlet kernels and the Fejér kernels are defined by

$$
\begin{aligned}
\widehat{f}(n) & :=\int_{G_{m}} f \overline{\psi_{n}} d \mu, \\
D_{n} & :=\sum_{k=0}^{n-1} \psi_{k}, \quad K_{n}:=\frac{1}{n} \sum_{k=0}^{n} D_{n},
\end{aligned}
$$

$(n \in \mathbb{N})$ where $D_{0}=K_{0}=0$. It is known [1] that

$$
D_{M_{n}}= \begin{cases}M_{n}, & \text { if } x \in I_{n} \\ 0, & \text { otherwise. }\end{cases}
$$


The general logarithmic kernels are

$$
Q_{n}=\frac{1}{H_{n}} \sum_{k=1}^{n-1} q_{k} D_{k} \quad(n \in \mathbb{P})
$$

where $q_{k}:=\frac{1}{k}$ or $\frac{1}{n-k}\left(\right.$ where $\left.Q_{1}:=0\right)$ and

$$
H_{n}=\sum_{k=1}^{n-1} \frac{1}{k}
$$

(set $H_{1}:=1$ ) is the $n$th harmonic number (see below for more details).

Namely, for $q_{k}=\frac{1}{k}$ we get $Q_{n}=R_{n}$ the $n$th Riesz kernels and for $q_{k}=\frac{1}{n-k}$ we have $Q_{n}=N_{n}$ the $n$th Nörlund kernels, respectively. That is,

$$
R_{n}=\frac{1}{H_{n}} \sum_{k=1}^{n-1} \frac{D_{k}}{k}, \quad N_{n}=\frac{1}{H_{n}} \sum_{k=1}^{n-1} \frac{D_{k}}{n-k} \quad(n \in \mathbb{P}) .
$$

The most important distinction between these kernels is that in the Riesz case the rear members of the sum have greater weight than the fore ones. Nörlund kernels can be considered as a "reverse" of Riesz kernels.

The maximal function of the kernels $Q_{n}$ 's is defined by

$$
Q^{*}(x):=\sup _{n \in \mathbb{P}}\left|Q_{n}(x)\right| \quad\left(x \in G_{m}\right) .
$$

The partial sums of the Fourier series with respect to a function $f$ are

$$
S_{n}(f)=\sum_{k=0}^{n-1} \widehat{f}(k) \psi_{k} .
$$

It is easy to see that $S_{n}(f)=f * D_{n}$, where $*$ denotes the standard convolution.

As in general, $\ell^{1}$ is the space which contains the sequences $\left(a_{n}\right.$ : $n \in \mathbb{N}$ ) for which $\sum_{k=0}^{\infty}\left|a_{k}\right|<\infty$.

Let $Q_{n}=R_{n}$ or $N_{n}$ in any occurrences from this point. As we shall see that the problem is the next:

$$
\left\|Q^{*}\right\|_{1}:=\int_{G_{m}} Q^{*} d \mu=+\infty
$$

for any bounded Vilenkin group $G_{m}$. Thus, we would like to find some monotone increasing weight function $\alpha:[0,+\infty[\rightarrow[1,+\infty[$ such that the integral of weighted maximal kernel

$$
Q_{\alpha}^{*}(x)=\sup _{n \in \mathbb{P}} \frac{\left|Q_{n}(x)\right|}{\alpha(n)} \quad\left(x \in G_{m}\right)
$$

turn to be finite. That is, $\left\|Q_{\alpha}^{*}\right\|_{1} \leq c<\infty$. This problem was raised by Gát [4] for the Walsh-Dirichlet and Walsh-Kaczmarz-Dirichlet kernels and he solved it. The second author discussed the behavior of maximal weighted kernels of Walsh-Fejér and Walsh-Kaczmarz-Fejér kernels [6]. 
For Vilenkin systems Simon and the first author [5] investigated the behavior of Vilenkin-Dirichlet and Vilenkin-Fejér kernels in this special point of view. We give more details later.

For Walsh group Gát used the map $\alpha \circ \log$, we denote it by $\widetilde{\alpha}$.

\section{Main Results}

Now, we formulate the results of this paper.

Theorem 1. If $G_{m}$ is a bounded Vilenkin group, then there exist constants $c, C>0$ such that

$$
c \sum_{A=1}^{\infty} \frac{1}{A \alpha\left(M_{A}\right)} \leq\left\|R_{\alpha}^{*}\right\|_{1} \leq C \sum_{A=1}^{\infty} \frac{1}{A \alpha\left(M_{A}\right)} .
$$

We immediately have

Corollary 1. For any bounded Vilenkin group $R_{\alpha}^{*} \in L^{1}$ if and only if $\left(1 / A \alpha\left(M_{A}\right): A \in \mathbb{P}\right) \in \ell^{1}$.

Corollary 2. In Walsh case

$$
c \sum_{A=1}^{\infty} \frac{1}{A \alpha(A)} \leq\left\|R_{\widetilde{\alpha}}^{*}\right\|_{1} \leq C \sum_{A=1}^{\infty} \frac{1}{A \alpha(A)} .
$$

Theorem 2. If $G_{m}$ is a bounded Vilenkin group, then there exist constants $c, C>0$ such that

$$
c \sum_{A=1}^{\infty} \frac{1}{\alpha\left(M_{A}\right)} \leq\left\|N_{\alpha}^{*}\right\|_{1} \leq C \sum_{A=1}^{\infty} \frac{1}{\alpha\left(M_{A}\right)} .
$$

We immediately have the following Corollaries:

Corollary 3. For any bounded Vilenkin group $N_{\alpha}^{*} \in L^{1}$ if and only if $\left(1 / \alpha\left(M_{A}\right): A \in \mathbb{P}\right) \in \ell^{1}$.

Corollary 4. In Walsh case

$$
c \sum_{A=1}^{\infty} \frac{1}{\alpha(A)} \leq\left\|N_{\widetilde{\alpha}}^{*}\right\|_{1} \leq C \sum_{A=1}^{\infty} \frac{1}{\alpha(A)} .
$$

This means that the behavior of the maximal function of weighted Nörlund logarithmic kernels is worse than the behavior of the maximal function of weighted Riesz logarithmic kernels. This means that the two summation method are far away from each other.

To gather informations on maximal functions of weighted kernels, we give a comprehensive tabular. For details, see $[4,5,6]$. 


\begin{tabular}{|l|c|}
\hline Kernel type & Condition on $\alpha$ \\
\hline Vilenkin-Dirichlet & $\frac{\ln \left(m_{A}\right)}{\alpha\left(M_{A}\right)} \in \ell^{1} \Leftrightarrow D_{\alpha}^{*} \in L^{1}$ \\
\hline Vilenkin-Fejér & $\frac{\ln \left(m_{A}\right)}{\alpha\left(M_{A}\right)} \in \ell^{1} \Leftrightarrow K_{\alpha}^{*} \in L^{1}$ \\
\hline Bounded Vilenkin-Riesz & $\frac{1}{A \alpha\left(M_{A}\right)} \in \ell^{1} \Leftrightarrow R_{\alpha}^{*} \in L^{1}$ \\
\hline Bounded Vilenkin-Nörlund & $\frac{1}{\alpha\left(M_{A}\right)} \in \ell^{1} \Leftrightarrow N_{\alpha}^{*} \in L^{1}$ \\
\hline Walsh-Kaczmarz-Dirichlet & $\frac{A}{\alpha(A)} \in \ell^{1} \Leftrightarrow D_{\widetilde{\alpha}}^{\kappa, *} \in L^{1}$ \\
\hline Walsh-Kaczmarz-Fejér & $\frac{1}{\alpha(A)} \in \ell^{1} \Leftrightarrow K_{\widetilde{\alpha}}^{\kappa, *} \in L^{1}$ \\
\hline
\end{tabular}

As our theorems and earlier results show that, the best summability method have to be the Riesz's method.

We need some results on harmonic numbers and their sums. Here we recall some results described in [2]. First of all, the next estimation is valid:

$$
\frac{1}{2 n}+\ln (n-1)+\gamma<H_{n}<\frac{1}{2(n-1)}+\ln (n-1)+\gamma \quad(n \in \mathbb{N}),
$$

where $\gamma=0.5772 \ldots$ is the Euler-Mascheroni constant.

Second order harmonic numbers (also called hyperharmonic numbers) are well-known notions in number theory.

$$
H_{n}^{(2)}=\sum_{k=2}^{n} H_{k}
$$

There is a useful connection between harmonic and hyperharmonic numbers:

$$
H_{n}^{(2)}=n\left(H_{n+1}-1\right) .
$$

We remark that one can define higher order harmonic numbers also. They appear in combinatoric number theory, too.

\section{ProOfs}

During the proofs the constants are not necessarily the same in several occurrences.

Proof of Theorem 1: First of all, we show that $\left\|R^{*}\right\|_{1}=+\infty$. For every $x \in I_{A} \backslash I_{A+1}$ we have that $D_{k}(x)=k$ for $1 \leq k<M_{A+1}$ and

$$
R_{M_{A}}(x)=\frac{1}{H_{M_{A}}} \sum_{k=1}^{M_{A}-1} \frac{D_{k}(x)}{k}=\frac{1}{H_{M_{A}}}\left(M_{A}-1\right) \geq \frac{1}{2} \frac{M_{A}}{H_{M_{A}}} .
$$

Now, we deduce the infiniteness of $\left\|R^{*}\right\|_{1}$. To do this we decompose the set $G_{m}$ as a disjoint union

$$
G_{m}=\bigcup_{A=0}^{\infty}\left(I_{A} \backslash I_{A+1}\right)
$$

and we write that 


$$
\begin{aligned}
\left\|R^{*}\right\|_{1} & =\int_{G_{m}} \sup _{n \in \mathbb{P}}\left|R_{n}(x)\right| d \mu(x)=\sum_{A=0}^{\infty} \int_{I_{A} \backslash I_{A+1}} \sup _{n \in \mathbb{P}}\left|R_{n}(x)\right| d \mu(x) \\
& \geq \sum_{A=0}^{\infty} \int_{I_{A} \backslash I_{A+1}}\left|R_{M_{A}}(x)\right| d \mu(x) \geq \frac{1}{2} \sum_{A=0}^{\infty} \int_{I_{A} \backslash I_{A+1}} \frac{M_{A}}{H_{M_{A}}} d \mu \\
& \geq \frac{1}{2} \sum_{A=0}^{\infty} \frac{1}{M_{A+1}} \frac{M_{A}}{H_{M_{A}}} \geq \frac{1}{2 m_{*}} \sum_{A=0}^{\infty} \frac{1}{H_{M_{A}}} .
\end{aligned}
$$

Moreover, it is simple to show that

$$
c A \leq c \ln 2^{A} \leq c \ln \left(M_{A}\right) \leq H_{M_{A}} \leq C \ln \left(M_{A}\right) \leq C \ln \left(m_{*}^{A}\right) \leq C A .
$$

This immediately gives our statement.

To get the lower estimation for the weighted Riesz kernel, let us follow the steps in the proof of infiniteness. We get

$$
\begin{aligned}
\left\|R_{\alpha}^{*}\right\|_{1} & =\int_{G_{m}} \sup _{n \in \mathbb{P}} \frac{\left|R_{n}(x)\right|}{\alpha(n)} d \mu(x) \geq \sum_{A=1}^{\infty} \int_{I_{A} \backslash I_{A+1}} \frac{\left|R_{M_{A}}(x)\right|}{\alpha\left(M_{A}\right)} d \mu(x) \\
& \geq \frac{1}{2} \sum_{A=1}^{\infty} \frac{1}{M_{A+1}} \frac{M_{A}}{H_{M_{A}} \alpha\left(M_{A}\right)} \geq \frac{1}{2 m_{*}} \sum_{A=1}^{\infty} \frac{1}{H_{M_{A}} \alpha\left(M_{A}\right)} \\
& \geq c \sum_{A=1}^{\infty} \frac{1}{A \alpha\left(M_{A}\right)} .
\end{aligned}
$$

The upper bound estimation:

$$
\begin{aligned}
R_{\alpha}^{*}= & \sup _{n \in \mathbb{P}} \frac{\left|R_{n}\right|}{\alpha(n)} \leq \sup _{n \in \mathbb{P}} \frac{1}{H_{n} \alpha(n)}\left|\sum_{k=1}^{M_{|n|}-1} \frac{D_{k}}{k}\right|+\sup _{n \in \mathbb{P}} \frac{1}{H_{n} \alpha(n)}\left|\sum_{k=M_{|n|}}^{\mid n_{|n|} M_{|n|}-1} \frac{D_{k}}{k}\right| \\
& +\sup _{n \in \mathbb{P}} \frac{1}{H_{n} \alpha(n)}\left|\sum_{k=n_{|n|} M_{|n|}}^{n-1} \frac{D_{k}}{k}\right|=: S^{1}+S^{2}+S^{3} .
\end{aligned}
$$

It is well-known [1] that

$$
D_{n_{A} M_{A}+j}=D_{n_{A} M_{A}}+\psi_{n_{A} M_{A}} D_{j}, \quad 0 \leq j<M_{A}, 0 \leq n_{A}<m_{A} .
$$


First, we discuss $S^{1}$ ( $S^{2}$ goes analogously).

$$
\begin{aligned}
S^{1} \leq & \sup _{n \in \mathbb{P}} \frac{1}{H_{n} \alpha(n)} \sum_{A=0}^{|n|-1}\left|\sum_{k=M_{A}}^{M_{A+1}-1} \frac{D_{k}}{k}\right| \\
\leq & \sup _{n \in \mathbb{P}} \sum_{A=0}^{|n|-1} \frac{1}{H_{M_{A}} \alpha\left(M_{A}\right)} \sum_{B=1}^{m_{A}-1}\left|\sum_{k=B M_{A}}^{(B+1) M_{A}-1} \frac{D_{k} \mid}{k}\right| \\
\leq & \sup _{n \in \mathbb{P}} \sum_{A=0}^{|n|-1} \frac{1}{H_{M_{A}} \alpha\left(M_{A}\right)} \sum_{B=1}^{m_{A}-1}\left|\sum_{l=0}^{M_{A}-1} \frac{D_{B M_{A}+l}}{B M_{A}+l}\right| \\
\leq & \sum_{A=0}^{\infty} \frac{1}{H_{M_{A}} \alpha\left(M_{A}\right)} \sum_{B=1}^{m_{A}-1}\left|\sum_{l=0}^{M_{A}-1} \frac{D_{B M_{A}}}{B M_{A}+l}\right| \\
& +\sum_{A=0}^{\infty} \frac{1}{H_{M_{A}} \alpha\left(M_{A}\right)} \sum_{B=1}^{m_{A}-1}\left|\sum_{l=0}^{M_{A}-1} \frac{D_{l}}{B M_{A}+l}\right| \\
= & S^{1,1}+S^{1,2} .
\end{aligned}
$$

From equation (1) we have that $\left\|D_{B M_{A}}\right\|_{1} \leq c$ and

$$
\begin{aligned}
\left\|S^{1,1}\right\|_{1} & \leq \sum_{A=0}^{\infty} \frac{c}{H_{M_{A}} \alpha\left(M_{A}\right)} \sum_{B=1}^{m_{A}-1} \sum_{l=0}^{M_{A}-1} \frac{1}{B M_{A}+l} \\
& \leq c \sum_{A=0}^{\infty} \frac{\left(H_{m_{A} M_{A}}-H_{M_{A}-1}\right)}{H_{M_{A}} \alpha\left(M_{A}\right)} \\
& \leq c \sum_{A=0}^{\infty} \frac{\ln m_{A}}{H_{M_{A}} \alpha\left(M_{A}\right)} \leq c \sum_{A=1}^{\infty} \frac{1}{A \alpha\left(M_{A}\right)} .
\end{aligned}
$$

To discuss $S^{1,2}$ we use Abel's transformation.

$$
\begin{aligned}
\left|\sum_{l=0}^{M_{A}-1} \frac{D_{l}}{B M_{A}+l}\right| \leq & \left|\sum_{l=1}^{M_{A}-2}\left(\frac{1}{B M_{A}+l}-\frac{1}{B M_{A}+l+1}\right) l K_{l}\right| \\
& +\frac{M_{A}-1}{(B+1) M_{A}-1}\left|K_{M_{A}-1}\right| .
\end{aligned}
$$

There exists a $c>0$ such that $\left\|K_{j}\right\|_{1} \leq c$ for all $j \in \mathbb{P}$ (see [7]). Thus,

$$
\begin{aligned}
\left\|S^{1,2}\right\|_{1} & \leq \sum_{A=0}^{\infty} \frac{c}{H_{M_{A}} \alpha\left(M_{A}\right)} \sum_{B=1}^{m_{A}-1}\left(\sum_{l=1}^{M_{A}-2} \frac{1}{B M_{A}+l}+\frac{M_{A}-1}{(B+1) M_{A}-1}\right) \\
& \leq \sum_{A=0}^{\infty} \frac{c\left(H_{m_{A} M_{A}}-H_{M_{A}}+m_{A}\right)}{H_{M_{A}} \alpha\left(M_{A}\right)} \leq \sum_{A=1}^{\infty} \frac{c}{A \alpha\left(M_{A}\right)} .
\end{aligned}
$$


At last, we discuss $S^{3}$. By the help of equation (2) we write that

$$
\begin{aligned}
S^{3} & =\sup _{n \in \mathbb{P}} \frac{1}{H_{n} \alpha(n)}\left|\sum_{k=n|n|}^{n-1} \frac{D_{k} \mid}{k}\right| \\
\leq & \sum_{A=0}^{\infty} \frac{1}{H_{M_{A}} \alpha\left(M_{A}\right)} \sup _{|n|=A}\left|\sum_{k=n_{A} M_{A}}^{n-1} \frac{D_{k}}{k}\right| \\
\leq & \sum_{A=0}^{\infty} \frac{1}{H_{M_{A}} \alpha\left(M_{A}\right)} \sup _{|n|=A}\left|\sum_{l=0}^{n-n_{A} M_{A}-1} \frac{D_{n_{A} M_{A}+l}}{n_{A} M_{A}+l}\right| \\
\leq & \sum_{A=0}^{\infty} \frac{\mid D_{n_{A} M_{A} \mid}}{H_{M_{A}} \alpha\left(M_{A}\right)} \sup _{|n|=A}\left|\sum_{l=0}^{n-n_{A} M_{A}-1} \frac{1}{n_{A} M_{A}+l}\right| \\
& +\sum_{A=0}^{\infty} \frac{1}{H_{M_{A}} \alpha\left(M_{A}\right)} \sup _{|n|=A}\left|\sum_{l=0}^{n-n_{A} M_{A}-1} \frac{D_{l}}{n_{A} M_{A}+l}\right| \\
= & S^{3,1}+S^{3,2} .
\end{aligned}
$$

From the above written

$$
\left\|S^{3,1}\right\|_{1} \leq c \sum_{A=0}^{\infty} \frac{\left(H_{m_{A} M_{A}}-H_{M_{A}}\right)}{H_{M_{A}} \alpha\left(M_{A}\right)} \leq c \sum_{A=1}^{\infty} \frac{1}{A \alpha\left(M_{A}\right)} .
$$

Now, we use Abel's transformation again

$$
\begin{aligned}
\left|\sum_{l=0}^{n-n_{A} M_{A}-1} \frac{D_{l}}{n_{A} M_{A}+l}\right| \leq & \sum_{l=1}^{n-n_{A} M_{A}-2} \frac{1}{n_{A} M_{A}+l}\left|K_{l}\right| \\
& +\frac{n-n_{A} M_{A}-1}{n-1}\left|K_{n-n_{A} M_{A}-1}\right| \\
\leq & \sum_{l=1}^{M_{A}-1} \frac{1}{n_{A} M_{A}+l}\left|K_{l}\right|+\frac{n-n_{A} M_{A}-1}{n-1}\left|K_{n-n_{A} M_{A}-1}\right| .
\end{aligned}
$$

This yields (by $\left\|K_{n}\right\|_{1} \leq c$ for all $n$ )

$$
\left\|S^{3,2}\right\|_{1} \leq \sum_{A=1}^{\infty} \frac{c\left(H_{m_{A} M_{A}}-H_{M_{A}}\right)+\left\|\sup _{|n|=A} \frac{n-n_{A} M_{A}-1}{n-1}\left|K_{n-n_{A} M_{A}-1}\right|\right\|_{1}}{A \alpha\left(M_{A}\right)} \text {. }
$$

We will show that there exists a $c>0$ such that $\| \sup _{|n|=A} \frac{n-n_{A} M_{A}-1}{n-1}$ $\left|K_{n-n_{A} M_{A}-1}\right| \|_{1} \leq c$, which immediately gives that

$$
\left\|S^{3,2}\right\|_{1} \leq c \sum_{A=1}^{\infty} \frac{1}{A \alpha\left(M_{A}\right)} .
$$

Let $n \in \mathbb{N}$ then (see [7])

$$
n\left|K_{n}\right| \leq c \sum_{j=0}^{|n|} M_{j} \sum_{i=j}^{|n|} \sum_{l=0}^{m_{j}-1} D_{M_{i}}\left(.+l e_{j}\right)
$$


Thus,

$$
\begin{aligned}
& \sup _{|n|=A} \frac{n-n_{A} M_{A}-1}{n-1} \mid K_{n-n_{A} M_{A}-1} \mid \leq \\
& \leq \sup _{|n|=A} \frac{c}{n-1} \sum_{j=0}^{A-1} M_{j} \sum_{i=j}^{A-1} \sum_{l=0}^{m_{j}-1} D_{M_{i}}\left(.+l e_{j}\right) \\
& \leq \frac{c}{M_{A}} \sum_{j=0}^{A-1} M_{j} \sum_{i=j}^{A-1} \sum_{l=0}^{m_{j}-1} D_{M_{i}}\left(.+l e_{j}\right) .
\end{aligned}
$$

From this and equality (1) we have

$$
\left\|\sup _{|n|=A} \frac{n-n_{A} M_{A}-1}{n-1}\left|K_{n-n_{A} M_{A}-1}\right|\right\|_{1} \leq \frac{c}{M_{A}} \sum_{j=0}^{A-1} M_{j+1}(A-j) \leq c .
$$

For more details see [7].

This completes the proof of Theorem 1 .

Proof of Theorem 2: The main approach is same as in the previous case, but the individual steps are slightly different.

First, we discuss the sum $\sum_{k=1}^{n-1} \frac{k}{n-k}$. Set $s=n-k$, then

$$
\sum_{k=1}^{n-1} \frac{k}{n-k}=\sum_{s=1}^{n-1} \frac{n-s}{s} .
$$

Moreover,

$$
\begin{aligned}
H_{n}^{(2)} & =H_{2}+H_{3}+\cdots+H_{n}= \\
& =\frac{1}{1}+\left(\frac{1}{1}+\frac{1}{2}\right)+\cdots+\left(\frac{1}{1}+\frac{1}{2}+\cdots+\frac{1}{n-1}\right)=\sum_{k=1}^{n-1} \frac{n-k}{k} .
\end{aligned}
$$

That is,

$$
\sum_{k=1}^{n-1} \frac{k}{n-k}=H_{n}^{(2)}
$$

For all $x \in I_{A} \backslash I_{A+1}$ we have that $D_{k}(x)=k$ for $1 \leq k<M_{A+1}$ and

$$
N_{M_{A}}(x)=\frac{1}{H_{M_{A}}} \sum_{k=1}^{M_{A}-1} \frac{D_{k}(x)}{n-k}=\frac{1}{H_{M_{A}}} \sum_{k=1}^{M_{A}-1} \frac{k}{n-k}=\frac{H_{M_{A}}^{(2)}}{H_{M_{A}}} .
$$

$H_{n}^{(2)}=n\left(H_{n+1}-1\right)$ (see above and [2]) yields that

$$
N_{M_{A}}(x) \geq \frac{M_{A}\left(H_{M_{A}+1}-1\right)}{H_{M_{A}}}
$$

for all $x \in I_{A} \backslash I_{A+1}$.

It means that we are totally in the same situation as in the Riesz case. Namely,

$$
\left\|N^{*}\right\|_{1}=+\infty
$$


for all bounded Vilenkin group. But,

$$
\begin{aligned}
\left\|N_{\alpha}^{*}\right\|_{1} & \geq \sum_{A=1}^{\infty} \int_{I_{A} \backslash I_{A+1}} N_{\alpha}^{*} d \mu \geq \sum_{A=1}^{\infty} \int_{I_{A} \backslash I_{A+1}} \frac{N_{M_{A}}}{\alpha\left(M_{A}\right)} d \mu \\
& \geq c \sum_{A=1}^{\infty} \frac{M_{A}\left(H_{M_{A}+1}-1\right)}{H_{M_{A}} \alpha\left(M_{A}\right)} \frac{1}{M_{A}} \geq c \sum_{A=1}^{\infty} \frac{1}{\alpha\left(M_{A}\right)} .
\end{aligned}
$$

The upper bound estimation:

$$
\begin{aligned}
N_{\alpha}^{*} & =\sup _{n \in \mathbb{P}} \frac{\left|N_{n}\right|}{\alpha(n)}=\sup _{n \in \mathbb{P}} \frac{\frac{1}{H_{n}}\left|\sum_{k=1}^{n-1} \frac{D_{k}}{n-k}\right|}{\alpha(n)} \leq \sup _{n \in \mathbb{P}} \frac{\frac{1}{H_{n}} \sum_{k=1}^{n-1} \frac{\left|D_{k}\right|}{n-k}}{\alpha(n)} \leq \\
& \leq \sup _{n \in \mathbb{P}} \frac{1}{H_{n}} \sum_{k=1}^{n-1} \frac{\left|D_{k}\right|}{\alpha(k)(n-k)} \leq \sup _{n \in \mathbb{P}} \frac{1}{H_{n}} \sum_{k=1}^{n-1} \frac{\left|D_{\alpha}^{*}\right|}{n-k}=D_{\alpha}^{*} .
\end{aligned}
$$

Recalling the bounded version of the theorem in [5] on maximal function of weighted Vilenkin-Dirichlet kernels, the result comes immediately.

\section{REFERENCES}

[1] G.H. Agaev, N.Ja. Vilenkin, G.M. Dzhafarli and A.I. Rubinstein, Multiplicative systems of functions and harmonic analysis on 0-dimensional groups, Baku ELM (1981) (Russian).

[2] J. H. Conway, R. K. Guy, The book of numbers, New York: Springer-Verlag, p. 258-259 (1996).

[3] G. Gát, On $(C, 1)$ summability for Vilenkin-like systems, Studia Math., 144(2) (2001), p. 101-120.

[4] G. Gát, On the $L_{1}$ norm of the weighted maximal function of the WalshKaczmarz-Dirichlet kernels, Acta Acad. Acad. Paed. Agriensis, Sect. Math., 30(3) (2003), p. 55-65.

[5] I. Mezö and P. Simon, Integrals of weighted maximal kernels with respect to Vilenkin systems, Publ. Math. Debrecen 71(1-2) (2007), p. 57-65.

[6] K. Nagy, On the $L^{1}$ norm of the weighted maximal function of Fejér kernels with respect to theWalsh-Kaczmarz system, Journal of Inequalities in Pure and Applied Mathematics 9(1) (2008), p. 1-9.

[7] J. Pál and P. Simon, On a generalization of the concept of derivative, Acta Math. Acad. Sci. Hung. 29(1-2) (1977), p. 155-164.

[8] F. Schipp, W.R. Wade, P. Simon and J. Pál, Walsh series: an introduction to dyadic harmonic analysis, Adam Hilger, Bristol and New York, 1990.

[9] N.Ja. Vilenkin, On a class of complete orthonormal systems, Izv. Akad. Nauk. SSSR, Ser. Math. 11 (1947), p. 363-400 (Russian).

István Mező, Department of Applied Mathematics and Probability Theory, Faculty of Informatics, University of Debrecen, Hungary

E-mail address: mezo.istvan@inf.unideb.hu

Károly Nagy, Institute of Mathematics and Computer Sciences, ColLEge of Nyíregyháza, Hungary

E-mail address: nkaroly@nyf.hu 